\title{
Incidence of optic canal fracture in the traumatic optic neuropathy and its effect on the visual outcome
}

\author{
Wentao Yan, ${ }^{2}$ Yingbai Chen, ${ }^{3}$ Zhenbin Qian, ${ }^{2}$ Dinesh Selva, ${ }^{4}$ Daniel Pelaez, ${ }^{5}$ \\ Yunhai $\mathrm{Tu}^{2}{ }^{2}$ Wencan $\mathrm{Wu}^{1}$
}

${ }^{1}$ Department of Orbital and Oculoplastic Surgery, Eye Hospital of Wenzhou Medical University, Wenzhou, Zhejiang, PR China

${ }^{2}$ Department of Orbital and Oculoplastic Surgery, Eye Hospital of Wenzhou Medical University, Wenzhou, PR China ${ }^{3}$ Department of Ophthalmology, Shanghai Jiao Tong University School of Medicine Affiliated Renji Hospital, Shanghai, China ${ }^{4}$ Discipline of Ophthalmology and Visual Sciences, University of Adelaide and South Australian Institute of Ophthalmology, Adelaide, Australia

${ }^{5}$ Bascom Palmer Eye Institute, University of Miami Miller School of Medicine, Miami, Florida, USA

\section{Correspondence to} Professor Wencan Wu, Department of Orbital and Oculoplastic Surgery, Eye Hospital of Wenzhou Medical University, No. 270 Xueyuan Xi Road, Wenzhou, Zhejiang 325027, P. R. China; wuwencan118@163.com

$W Y$ and $Y C$ are co-first authors and contributed equally.

Received 28 October 2015 Revised 29 April 2016 Accepted 12 May 2016 Published Online First 6 June 2016

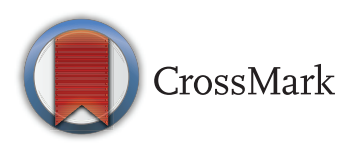

To cite: Yan W, Chen $Y$, Qian Z, et al. Br J

Ophthalmol 2017;101:261267.
ABSTRACT

Objective To correlate the intraoperative endoscopic findings with high-resolution CT (HRCT) for the diagnosis of optic canal fractures (OCF). To compare the visual outcome of patients with different types of OCF and without.

Design A retrospective, comparative case series. Participants 1275 consecutive patients (1275 eyes) with indirect traumatic optic neuropathy (TON).

Methods Altogether, 1275 patients who underwent endoscopic transethmoid optic canal decompression (ETOCD) for unilateral indirect TON were reviewed from 1 October 2006 to 30 September 2014. HRCT performed prior to surgery were compared with findings during surgery. The visual outcomes before and after surgery were also compared.

Main outcome measures The presence and type of OCF detected by HRCT and during surgery; visual acuity prior to surgery and at 3 months after surgery.

Results A total of 1275 patients (1275 eyes) were included, with 708 patients that had OCF visible on HRCT image. During surgery, an additional 187 (20.9\%) patients with OCF were noticed. Among these, $136 \mathrm{had}$ undisplaced fractures, most of which were linear intracanalicular fractures. The initial visual acuity of patients with OCF was worse than that of patients without OCF $(p<0.01)$. However, no statistical difference existed in the final visual acuity at 3 months after surgery (>0.05). Significant statistical difference of surgical efficacy existed between all the patients with OCF and without OCF $(p<0.001)$.

Conclusions Among patients with OCF, 20.9\% were not detected by HRCT. Patients with OCF had a worse initial visual acuity than those without OCF. There was no significant difference in final visual acuity after ETOCD.

\section{INTRODUCTION}

The most common form of traumatic optic neuropathy (TON) is indirect and results from concussive force to the head, especially the forehead. This impact is thought to transmit a shock wave to the optic canal, damaging the optic nerve. ${ }^{1-3}$ Therefore, the surrounding soft tissue and bony injuries of the optic canal are believed to be helpful to indirectly assess the degree of impact force on the optic nerve and may be used as a prognosis predictor of TON. ${ }^{4-12}$ There is inadequate evidence that the presence of optic canal fractures (OCF) is sufficient to shift the risk/benefit equation in favour of canal decompression, but early canal decompression is generally recommended when there is a displaced fragment impinging on the optic nerve. ${ }^{3} 11$ 13-15

The role of high-resolution CT (HRCT) in testing TON has been well established and is generally considered essential for detecting possible contributors to optic nerve injury, including retrobulbar haemorrhage, optic nerve oedema, intraorbital emphysema and OCF. ${ }^{4-11}{ }^{13}$ However, the actual incidence, position and orientation of $\mathrm{OCF}$ in indirect TON are uncertain. The reported incidence of OCF on CT scans varies considerably from 5\% to $92 \% .{ }^{17}{ }^{16}$ Based on direct endoscopic observation at the time of surgery in a few previous case series, OCF may exist even if it cannot be detected radiologically. ${ }^{14-15}$ 17-19

The effect of OCF on visual outcome is debatable, with a number of papers reporting orbital fractures as a poor predictor. ${ }^{20-23}$ Here, we compare the results of HRCT scans prior to surgery with the findings during surgery in a large group of patients with indirect TON. Our intention was to verify the reliability of HRCT in OCF diagnosis. Meanwhile, we reported the visual outcome of patients with different types of OCF.

\section{MATERIALS AND METHODS Patient selection}

All patients were admitted and underwent endoscopic transethmoid optic canal decompression (ETOCD) due to unilateral indirect TON from 1 October 2006 to 30 September 2014. They were identified from the hospital clinical databases at the Eye Hospital of Wenzhou Medical University, the Lishui Central Hospital, the Jinhua Central Hospital, Lihuili Hospital in Ningbo, Quzhou People's Hospital in Zhjiang province, Xiamen Eye Hospital in Fujian province, The Fourth Hospital of Shengyang in Liaoning province, and The Second People's Hospital of Zhengzhou city in Henan province. TON was diagnosed based on severe reduction or loss in visual acuity (VA) accompanied by a relatively afferent papillary defect after a closed head injury. TON could not be explained by any other causes. If necessary, visual field examination and visual evoked potential (VEP) were performed for diagnosis. ETOCD was performed on patients whose VA was no more than 20/100 with no improvement after 4-5 days of conservative treatment. The conservative treatment included intravenous administration of methylprednisolone of $500-1000 \mathrm{mg}$ for the first 2 days and half dosage for the following days, intramuscular injection of mouse-derived nerve growth factor 
(NGF) (Staidson (Beijing) Biopharmaceuticals Co.) of $30 \mu \mathrm{g} /$ day for 4-5 days, etc. In extreme cases that patients had no light perception (NLP) immediately after trauma without any improvement after methylprednisolone administration for 2 days, ETOCD was recommended to be performed immediately. All patients were examined by neurologists to make sure the safety for ETOCD. Patients with bilateral TON were excluded, because they were usually so badly injured that they were unable to cooperate with the ophthalmic examinations or not suitable for ETOCD. Patients with impaired consciousness and patients under the age of 16 were ruled out because of lack of cooperation and unavailable informed consent by themselves. Patients without detected VEP were not admitted for this procedure, considering poor therapeutic outcome for severely impaired retinofugal pathway. In order to avoid the interference of bone healing on the results, patients who had ETOCD later than the 14th day after the injury were also excluded. Written consent was obtained from all patients before surgery in accordance with the institutional review board's policies. The research followed the principles of the Declaration of Helsinki. This research was approved by the ethics committees of all eight hospitals involved.

\section{Image interpretation}

Orbital HRCT was performed for each patient at the time of admission with a 40-section or 64-section CT system (GE, Milwaukee, Wisconsin, USA; or Philips, Amsterdam, China) (figure 1). A standard protocol was used similar to that described by Lee et al. ${ }^{9}$ Axial images, $1 \mathrm{~mm}$ thick, were made parallel to the optic nerve in a line from the inferior orbital rim to the upper pinna. Coronal images, $3 \mathrm{~mm}$ thick, were made from the globes to the dorsum sella, with the sections perpendicular to the canthomeatal line. In patients unable to hyperextend the neck, coronal reformations from the axial images were performed. For the patients at the Eye Hospital of Wenzhou Medical University, the bone and soft tissue windows of all of the axial and coronal CT scans were carefully reviewed and analysed together by an ophthalmological clinician (Dr Yunhai Tu) and two experienced radiologists with expertise in head and neck imaging for consensus prior to surgery. For patients at the other seven institutes, all clinical data were sent to Dr Yunhai Tu by email for consultation, and the CT scan of each patient was prospectively reviewed by Dr Yunhai $\mathrm{Tu}$ and the two experienced radiologists for consensus before operation.

We recorded the age of the patient, Snellen's VA prior to surgery and the final VA at 3 months after surgery, time interval between injury and surgery, HRCT findings and the surgical findings. Colour vision, visual field defects and VEP were not consistently documented in the medical records and were not included in the study. Usually, patients were followed up to 3 months at these eight institutions. For a few patients unable to come, we recommended them to local community hospitals for basic post-op examinations and collected outcome from them by telephone at 3 months after surgery. For the convenience of data analysis and comparison, Snellen's VA was transferred to $\operatorname{logMAR}$ units. $^{21} 24-26$ We applied the formula of Chen et al ${ }^{25}$ to define the percentage of improvement degree of VA (IDVA):

postoperative log MAR - preoperative log MAR/(0.12

$$
\text { * - preoperative log MAR) }
$$

*where log MAR $0.12(20 / 15)$ is considered perfect vision.
If the IDVA decreased or remained the same after surgery, the surgery was considered to be ineffective. If the IDVA increased no more than $50 \%$, the surgery was considered to be moderate effective, and if the IDVA increased more than 50\%, the surgery was considered to be very effective.

\section{Surgical procedure of ETOCD}

All of the procedures were performed under general anaesthesia by the surgeon (Dr Wencan Wu) as previously described. ${ }^{27-29}$ The orbital lamina papyracea was exposed via a standardised ethmoidectomy under direct visualisation, with a $4 \mathrm{~mm} 45^{\circ}$ transnasal endoscope (KARL STORZ, Tuttlingen, Germany), prior to opening the sphenoid sinus. The bulge caused by the optic canal and internal carotid artery was identified in the lateral wall of the sphenoidal sinus. After meticulous removal of the sinus mucosa, the area was inspected for OCF, fractures of the lesser wing of the sphenoid bone, anterior skull base fractures and/or cerebrospinal rhinorrhoea (figure 2A). The lesser wing of the sphenoidal bone and the medial wall of the optic canal, which spans from the orbital aperture to the cranial cavity, were thinned with a microdrill and removed with a microcurette (figure $2 \mathrm{~B}, \mathrm{C}$ ). Then, the periorbita of the orbital apex, annulus of Zinn and the optic nerve sheath were incised with a sharp $9^{\#}$ MVR knife (figure 2D). Finally, at the end of the surgery, the operating field of the optic canal was covered by a piece of sterile gelatine sponge that was immersed in dexamethasone $(5 \mathrm{mg} / 2 \mathrm{~mL})$ and mouse-derived NGF $(30 \mu \mathrm{g} / \mathrm{mL})$ (Staidson (Beijing) Biopharmaceuticals Co.).

Postoperative care included intravenous methylprednisolone, $30 \mathrm{mg} / \mathrm{kg} /$ day for 3 days and intravenous ceftriaxone for 5 days. At the third postoperative day, the dexamethasone and mousederived NGF were locally administered after removal of the gelatine, and this procedure was done every 2 days for a total of five times.

\section{Statistical analysis}

Statistical analyses were performed using SPSS V.20.0 software (SPSS, Chicago, Illinois, USA). Univariate analysis was performed by Pearson's $\chi^{2}$ tests for discrete variables, and paired sample $t$ test or independent samples t test and one-way analysis of variance for continuous variables. Results were considered significant at $\mathrm{p}<0.05$ and reported as mean $\pm \mathrm{SD}$.

\section{RESULTS}

\section{Baseline characteristics and periorbital injury}

Altogether, 1275 consecutive patients (1275 eyes) were included, with a male to female ratio of 3.7 to 1 . The age of the patients ranged from 16 to 50 years old, with a mean of 29.8 \pm 7.3 years old. The time interval from injury to surgical intervention ranged from 1 to 14 days, with a mean of $10 \pm 2$ days. Road traffic accident was the most common cause of injury (659 patients), followed by blunt assault (261 patients), work-related injuries (226 patients) and fall from a height (131 patients). Multiple facial fractures were present in 517 patients. Intracerebral haemorrhage was noticed in 217 patients. Concurrent HRCT findings related to intraorbital soft tissue injuries included extraconal haematoma (255 patients), intraconal haematoma (149 patients), intraconal emphysema (132 patients) and intrasheath haematoma along the optic nerve (53 patients).

\section{Differences between images and surgical findings}

HRCT images prior to surgery showed that 708 patients (55.5\%) had visible OCF, of which $264(20.7 \%)$ were displaced 
Figure 1 Images from Philips Brilliance 64-channel CT scanner; standard orbital high-resolution CT scans performed for patients with traumatic optic neuropathy. (A) Axial images, $1 \mathrm{~mm}$ thick, parallel to the optic nerve in a line from the inferior orbital rim to the upper pinna. (B) Axial CT scan shows significant displaced intracanalicular fracture without impingement. (C) Coronal CT scan shows displaced intracanalicular fracture and anterior cranial base fracture (white arrows: fractures).
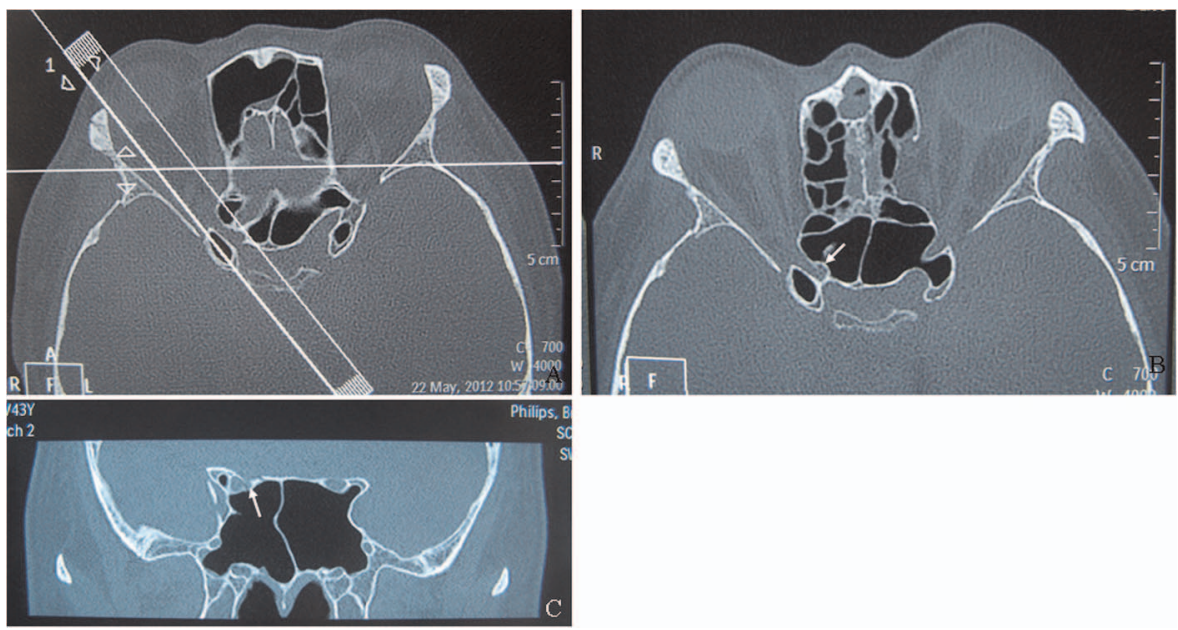

OCF with impingement on the optic nerve. During surgery, OCF was detected in 895 patients (70.2\%) (figure 3A-C), including 618 cases of single fracture and 277 cases of multiple fractures. The remaining 380 patients had no OCF confirmed by surgery.

According to the exact location of fractures, OCF could be divided into four types, including intracanalicular fractures (365 patients), fractures in close proximity to the optic nerve's entry to the orbit (168 patients), fracture's proximity to the cranial opening of the canal (92 patients) and fractures involving most parts of the optic canal (270 patients).

OCF could also be classified as displaced OCF with impingement on optic nerve (264 patients) (figure 3A), displaced OCF without impingement on optic nerve (425 patients) (figure $3 \mathrm{~B}$ ) and OCF without displacement (206 patients) (figure 3C) (table 1). Among the 264 patients having displaced OCF with impingement, all identified on HRCT scans, 39 patients had fragments penetrating into the optic nerve. Among the 425 patients having displaced OCF without impingement, 51 patients (12\%) had slightly displaced comminuted fractures of the thin canal that were not evident on the HRCT scans (figure 4A). Among the 206 patients having OCF without displacement, 136 patients (66\%) were negative on the HRCT scans. These 136 cases were further confirmed of OCF during surgery, of which 115 cases were linear intracanalicular fractures without displacement (figure 4B) and 21 cases were OCF located at the junction of the optic nerve's entry to the orbit. Among these 21 cases, 6 cases were accompanied by multiple fracture of the lesser wing of the sphenoid bone of the orbital apex; 11 cases were accompanied by congenital abnormalities of the posterior ethmoid sinus and/or sphenoid sinus including Onodi cell extended upward to the sphenoidal sinus; 4 cases were accompanied by periorbital gas cell located in the orbital apex due to excessive pneumatisation of the sphenoidal sinus (figure 4C).

\section{Correlations of OCF with VA}

In this case series, 87 patients had NLP prior to surgery, among whom 37 had displaced OCF with impingement, 18 patients had displaced OCF without impingement, 9 patients had OCF
Figure 2 Procedure of endoscopic transethmoid optic canal decompression for a case with traumatic optic neuropathy. (A) Optic canal fractures (OCF) observed to be displaced without impingement on the optic nerve. (B) An optic canal was meticulously thinned with a microcurette. (C) The bone of the canal including the fragment was carefully removed with a small sickle knife. (D) Sheath of the optic canal was incised with a sharp $9^{\#}$ MVR knife (black arrow: OCF).
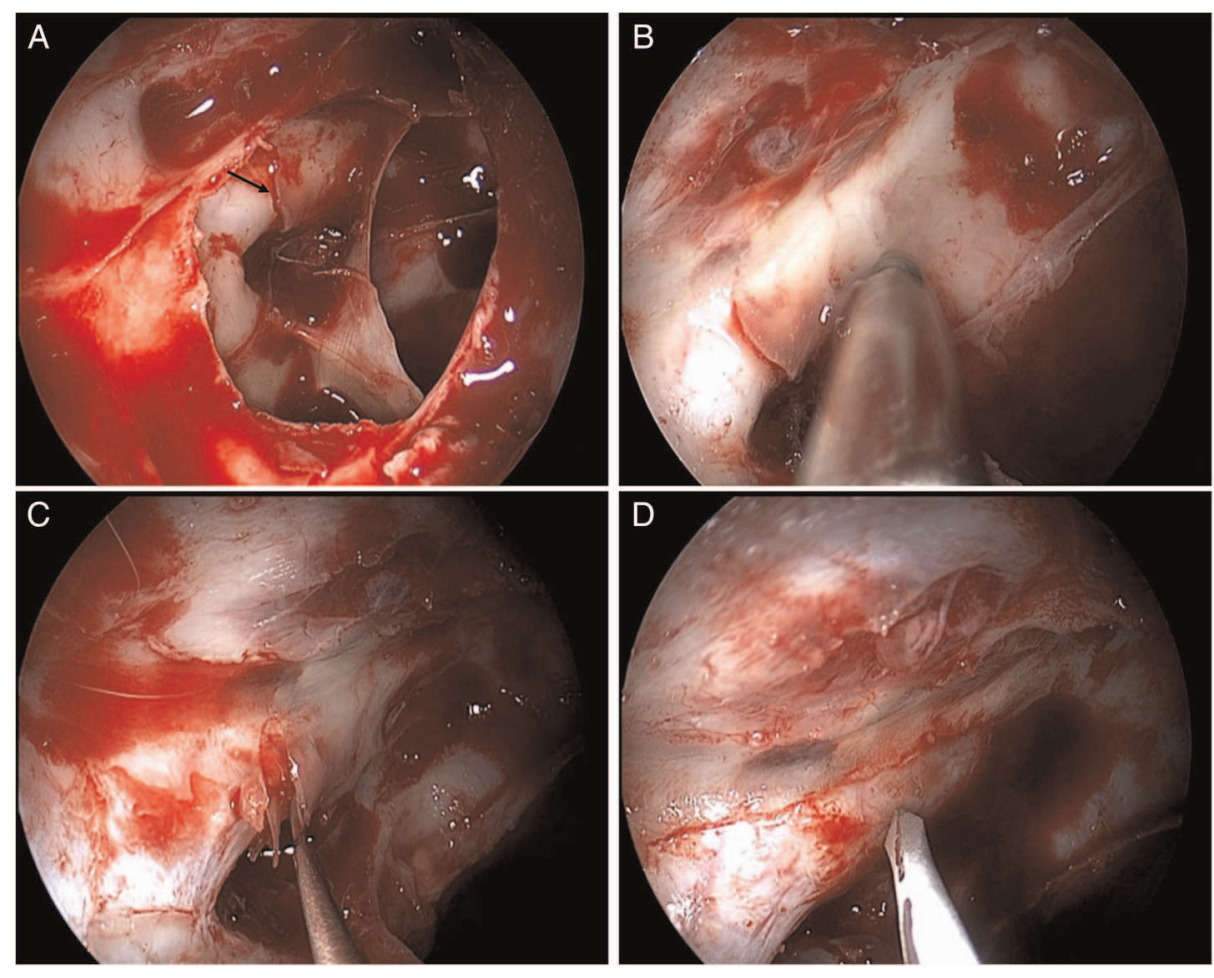

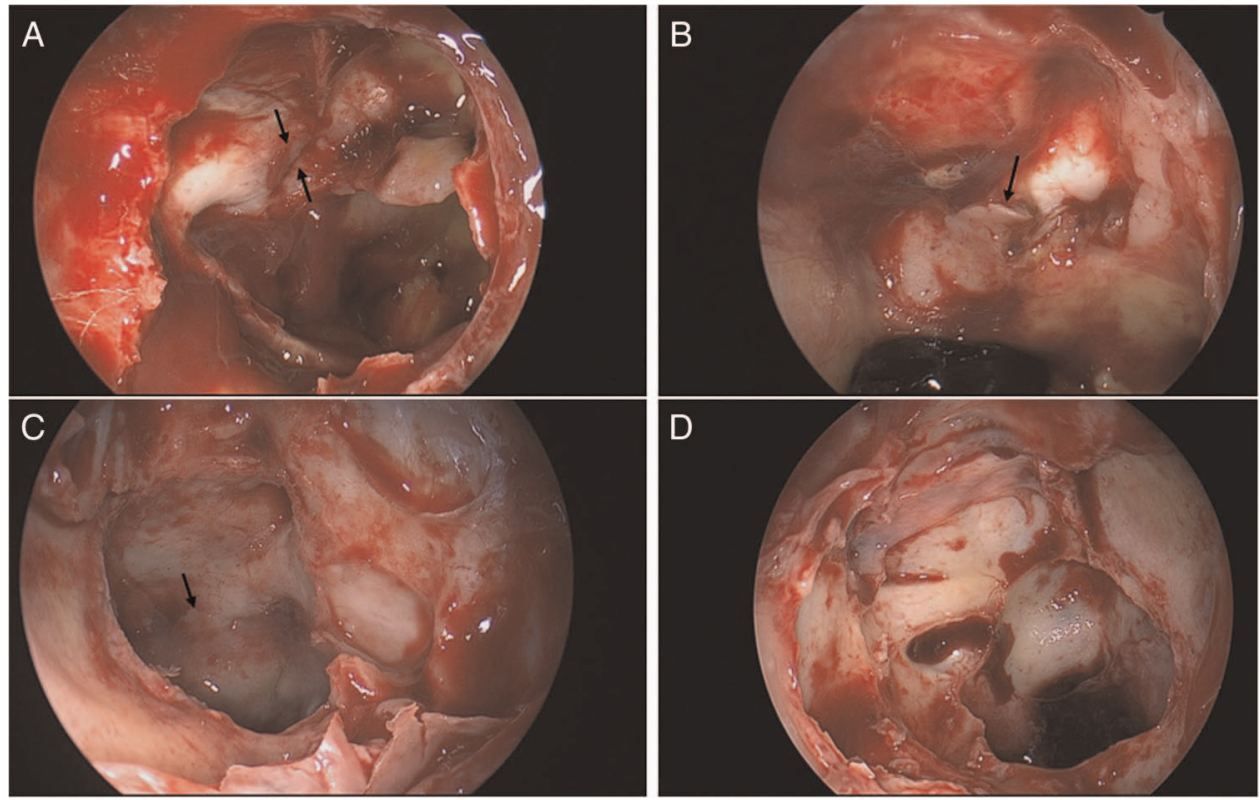

Figure 3 Different types of optic canal fractures (OCF) visible on high-resolution CT scans and confirmed at the time of surgery. (A) The fragments of the OCF were observed significantly displaced, with impingement on the optic nerve. (B) The fragments of the OCF were displaced significantly, without impingement on the optic nerve. (C) OCF without displacement. (D) The optic canal without fracture (black arrow: OCF).

without displacement and 23 patients did not have OCF. The initial VA of patients with OCF was $-2.42 \pm 1.06$, worse than $-2.06 \pm 1.09$ of patients without OCF $(\mathrm{t}=-5.509, \mathrm{p}<0.001)$. Furthermore, the initial VA in patients having displaced OCF with or without impingement was respectively worse than that of patients having no OCF $(\mathrm{p}<0.01)$. However, there was no statistical difference between patients having OCF without displacement and patients having no OCF. There was no statistical difference of initial VA between patients having displaced OCF without impingement and patients having OCF without displacement $(p>0.05)$. Also, no statistical difference of the final VA existed among different groups $(p>0.05)$. Compared with initial VA, the final VA at 3 months of all groups was improved significantly $(p<0.001)$ (figure 5$)$. This was consistent with the results of generalised linear model analysis adjusted by gender, side, age, initial VA and the time interval between the trauma and surgery.

According to IDVA, the patients were divided into three groups with different surgical efficacies, which were ineffective, moderate effective and very effective. Significant statistical difference of surgical efficacy existed between all the patients with OCF and without OCF $(\mathrm{p}<0.001)$ (table 2). Multiple

Table 1 Comparison of OCF diagnosed during surgery with HRCT findings prior to surgery

\begin{tabular}{llll}
\hline & $\begin{array}{l}\text { Orbital } \\
\text { HRCT }\end{array}$ & $\begin{array}{l}\text { Endoscopic } \\
\text { view }\end{array}$ & $\begin{array}{l}\text { Missing rate } \\
(\%)\end{array}$ \\
\hline $\begin{array}{l}\text { Displaced OCF with } \\
\text { impingement }\end{array}$ & 264 & 264 & 0 \\
$\begin{array}{l}\text { Displaced OCF without } \\
\text { impingement }\end{array}$ & 374 & 425 & $51(12 \%)$ \\
$\begin{array}{l}\text { OCF without displacement } \\
\text { No OCF }\end{array}$ & 70 & 206 & $136(66 \%)$ \\
\hline
\end{tabular}

Missing rate=(Number of patients missed out by HRCT scans)/(Number of patients confirmed during surgery).

HRCT, high-resolution computed topography; OCF, optic canal fracture. comparisons using $\chi^{2}$ test indicated that significant statistical difference of surgical efficacy existed among different groups $(\mathrm{p}<0.001)$, except for patients having displaced OCF with impingement versus patients having OCF without displacement and patients having displaced OCF without impingement versus patients without OCF ( $p>0.05)$ (figure 6).

Fifteen patients experienced cerebrospinal leakage after surgery which was repaired uneventfully. Several patients presented with nasal insignificant haemorrhage. Other significant complications were not observed, such as severe VA deterioration, chronic sinusitis, cranial infection or massive haemorrhage due to injury of carvernous sinus or internal carotid artery.

\section{DISCUSSION}

The role of CT in orbital trauma has been well established, and OCF has been reported to be optimally visualised with thin

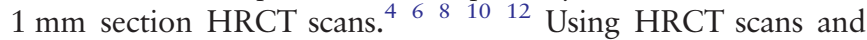
reformatted images of the optic canal region, Seiff et al detected six patients having OCF out of nine patients with sudden blindness resulting from indirect TON. Dietrich et al reported that among 10 patients with TON, 6 cases had OCF diagnosed via HRCT scans and another 3 cases that had orbital apex fractures negative on HRCT scans were confirmed during surgery. In this large-scale study, HRCT scans showed that 708 patients out of 1275 patients with TON (55.5\%) had OCF. All of these cases were intraoperatively confirmed under endoscopy. During surgery, an additional 187 patients were noticed to have OCF not visible on HRCT scans. The missing rate of OCF (not detected by HRCT scans) was 20.9\% (187/895). Among these 187 missing cases, 136 appeared to be non-displaced linear intracanalicular fractures and 51 appeared to be slightly displaced comminuted fractures without impingement. This finding is consistent with the results of a few case series which were reported earlier. Yang et $a l^{14}$ reported that among 96 patients of TON, HRCT scans revealed only 52 OCF cases, and another 10 OCF cases were further confirmed of medial wall fractures of the optic canal intraoperatively. Thus, the missing rate of fractures by HRCT scans was 16.1\% (10/62 patients). 

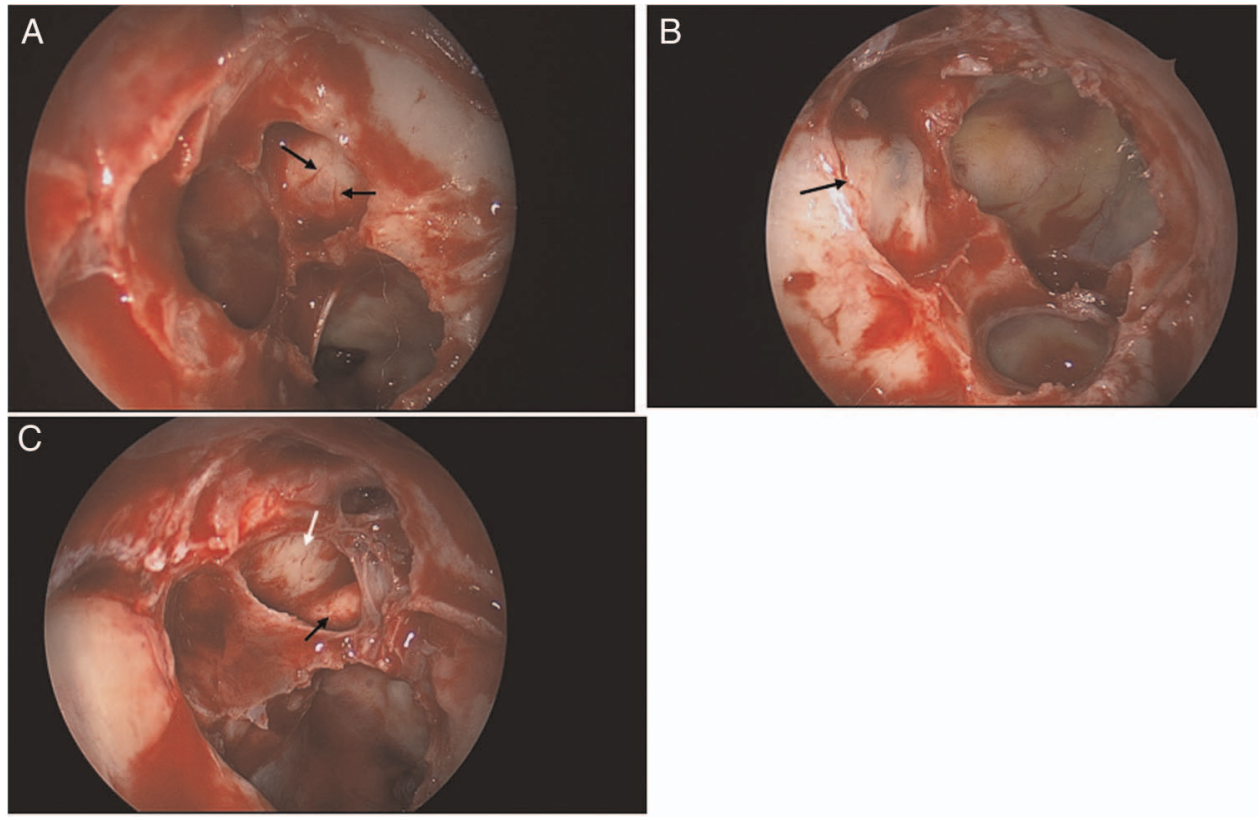

Figure 4 Optic canal fractures invisible on the high-resolution CT scans. (A) Comminuted fracture of the thin optic canal, though minimally displaced but without impingement. (B) Undisplaced linear intracanalicular fracture. (C) A minimally displaced fracture of the optic canal that passed through an abnormal small Onodi cell between the posterior ethmoidal sinus and the sphenoid sinus; its surrounding bones were thick and abnormal (black arrow: fractures; white arrow: Onodi cell).

Imachi $^{15}$ also reported 16 HRCT-undetected OCF cases out of 80 cases with TON, making missing rate 20\%. Gupta et al ${ }^{17}$ reported an even poor correlation between CT and operative findings, showing 7 OCF cases visible on CT and 13 OCF cases detected during surgery. Wohlrab et $a l^{18}$ reported a high falsenegative rate that only one OCF was detected by CT while eight were found intraoperatively. Along with our large-scale study, these reports clearly demonstrate that OCF may still exist even if it comes clear on CT image. So, attention should be paid to the reliability of radiographic evidence for diagnosis of OCF when assessing patients with sudden vision loss after head trauma.

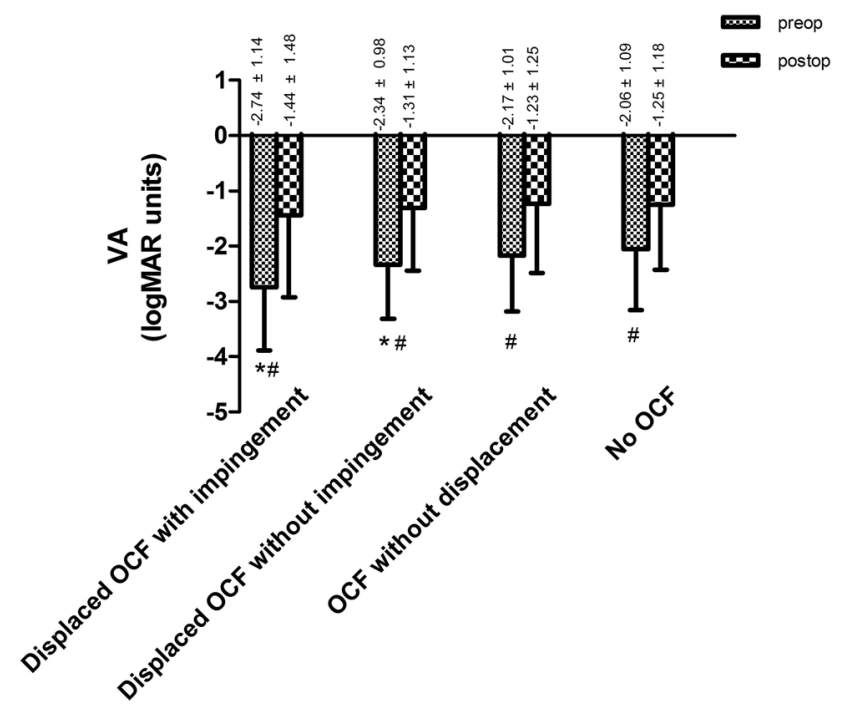

Figure 5 Visual acuity (VA) of different types of optic canal fractures (OCF) before and after surgery. ${ }^{*} p<0.01$ versus patients without OCF using one-way analysis of variance. $\# p<0.001$ versus the final VA at 3 months after the surgery using paired-samples t test.
The sensitivity of CT scans in OCF diagnosis relies on several influential factors, including the scanner machine, thickness of the slices taken, position and angle of the head, anatomical characteristics and experience of the clinician who interprets the scans. Based on this study, we believe that the causes of misdiagnosed OCF on HRCT scans may be attributed to the following three factors:

(1) Linear fractures are difficult to be detected. HRCT scanning is definitely accurate and reliable when the OCF is significantly displaced. However, for 206 patients without displacement, only 70 patients (34\%) were detected, and 136 patients $(66 \%)$ were missed out. The linear fractures seem to be the most difficult to detect with HRCT. As we can see, among the undetected 136 patients, 115 patients $(84.6 \%, 115 / 136)$ were intraoperatively observed to have linear intracanalicular fractures. In 1984, Unger $^{10}$ also proposed that undisplaced

Table 2 Surgical efficacy for patients with different types of OCF and without OCF

\begin{tabular}{|c|c|c|c|c|c|c|}
\hline \multirow[b]{2}{*}{ Group } & \multicolumn{2}{|c|}{ Ineffective } & \multicolumn{2}{|c|}{$\begin{array}{l}\text { Moderate } \\
\text { effective }\end{array}$} & \multicolumn{2}{|c|}{$\begin{array}{l}\text { Very } \\
\text { efficient }\end{array}$} \\
\hline & N & $\%$ & $\mathrm{~N}$ & $\%$ & N & $\%$ \\
\hline \multicolumn{7}{|l|}{ Kinds of OCF } \\
\hline Displaced OCF impingement & 82 & 31.1 & 7 & 2.7 & 175 & 66.3 \\
\hline $\begin{array}{l}\text { Displaced OCF with no } \\
\text { impingement }\end{array}$ & 59 & 13.9 & 122 & 28.7 & 244 & 57.4 \\
\hline OCF without displacement & 52 & 25.2 & 14 & 6.8 & 140 & 68.0 \\
\hline No OCF & 47 & 12.4 & 144 & 37.9 & 189 & 49.7 \\
\hline$\chi^{2}$ & & 166.416 & & & & \\
\hline$P$ & & $<0.001$ & & & & \\
\hline \multicolumn{7}{|c|}{$\begin{array}{l}\text { Moderate effective=IDVA no more than } 50 \% \text {; very efficient=IDVA } \geq 50 \% \text {; } \\
\text { ineffective=no improvement of VA after surgery. } \\
P=\text { =ifference of efficacy between patients with } 0 C F \text { and no OCF, using } \chi^{2} \text { test. } \\
\text { IDVA, improvement degree of VA; No OCF, patients who did not have OCF; OCF, optic } \\
\text { canal fracture: VA, visual acuity. }\end{array}$} \\
\hline
\end{tabular}


Figure 6 Multiple comparison of surgical efficacy for patients with different types of optic canal fractures (OCF) and without. Multiple comparison of efficacy using $\chi^{2}$ test and the correct $=0.05 / 6=0.083$; difference is existed if $\mathrm{p}<0.083$. between two groups. *Significant statistic difference existed

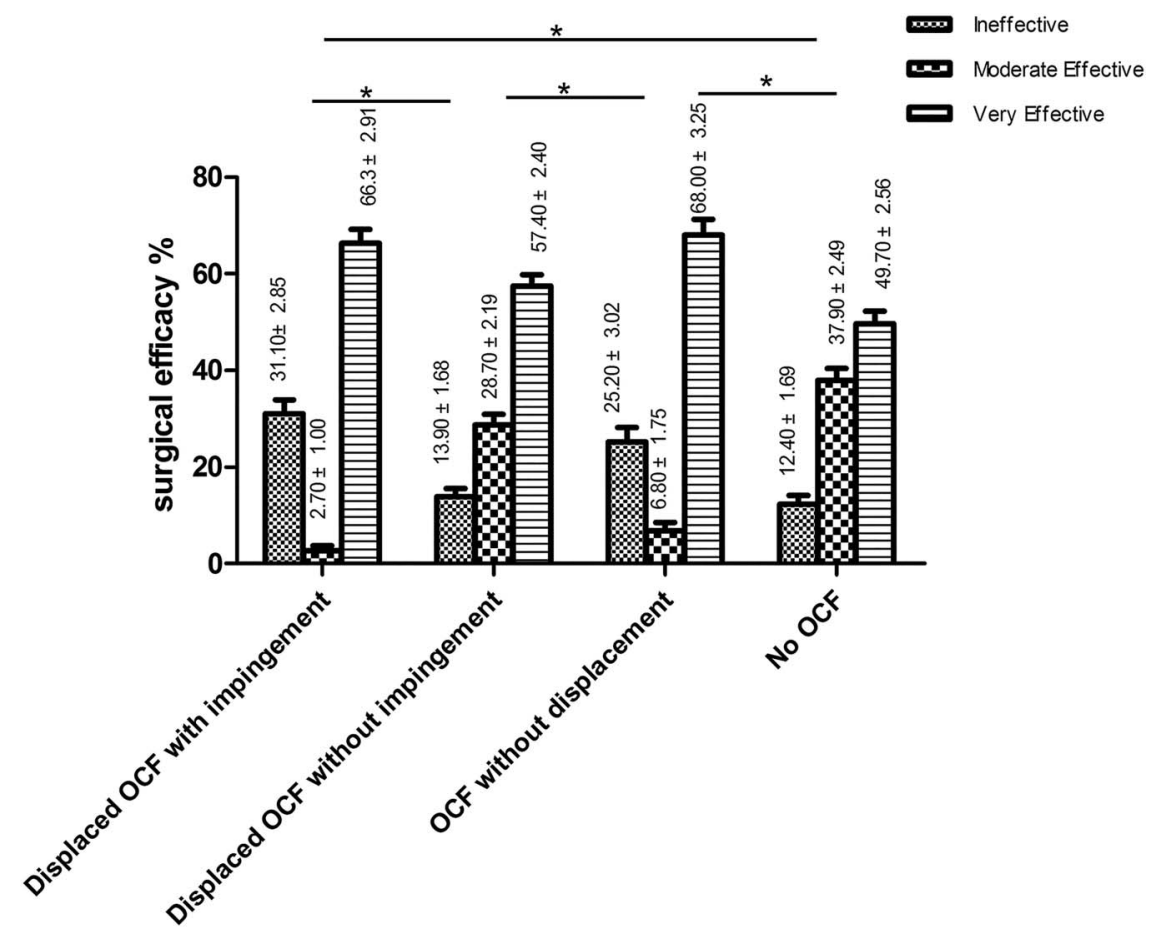

linear fractures were difficult to see on CT scans due to the thinness of the orbital walls, absence of displaced fragments and possible bony shadows.

(2) In fractures with slight displacement, the bone of the optic canal may be too thin to be identified on HRCT images. In this case series, among all the misdiagnosed cases, 51 were observed to have comminuted fractures of the optic canal with only tiny displacement. The slightly displaced fracture could be easily overlooked even during surgery if we did not carefully touch it with the tip of a small sickle knife.

(3) The optic canal and its adjacent structures may be anatomically abnormal, making detection of fractures much more difficult. In this study, there were 21 patients having a single fracture without impingement at the medial junction of the optic nerve's entry to the orbit; a special location could not show clearly on HRCT scans. We found that 6 patients had significantly anatomical deformation of the orbital apex, 11 patients had an Onodi cell and 4 patients had an obvious periorbital gas cell. We assumed that the fracture signals may be interfered by bony overlap of deformed fractures or the congenital anatomical abnormalities in the posterior ethmoid sinus and/or sphenoid sinus. Nowadays, some variations in paranasal sinus anatomy such as Onodi cell or periorbital gas cell can be detected by CT scans of paranasal sinuses. ${ }^{30} 31$ However, there is no specific data showing the correlation between these anatomical variations and OCF detection.

Until now, there has been no proof showing OCF as a prognosis factor. Based on this study, we assumed that OCF may be used as an indicator for VA prognosis. We found that the initial VA of patients with OCF is poorer than that of patients without OCF, and the initial VA of patients with displaced OCF was poorer than that of patients without OCF. This could be explained by the severity of the injury. The more serious da mage the patient experiences, the more severe type of OCF is likely to happen. Three months after surgery, VA improved in 702 out of 895 (78.4\%) patients with OCF and 333 out of 380 (87.6\%) patients without OCF. The VA improvement degree varies among patients with different kinds of OCF. There is significant difference of surgical efficacy between patients with OCF and without OCF. Furthermore, significant difference of surgical efficacy also exists among patients with different kinds of OCF and without. Our study establishes a correlation between the presence and type of optic canal fracture and the visual prognosis in TON.

It is controversial whether ETOCD will benefit the VA prognosis of patients with TON. Some reported that patients without any intervention showed a visual recovery rate of $40 \%-60 \%{ }^{16}$ However, Yu-Wai-Man et al ${ }^{16}$ suggest that the bone fragments are quite likely to transect retinal ganglion cell axons with irreversible damage, so that ETOCD may be necessary for patients at risk. In our study, the effective rate of surgery is $78.4 \%$ (702 out of 895 patients) for patients with OCF and 87.6\% (333 out of 380 patients) for patients without OCF. These data are quite optimistic. We hypothesise that for some patients with TON, visional recovery may be possible if appropriate stimuli were given to promote the retinal ganglion cells' survival, such as ETOCD. We believe a prospective interventional study would be necessary to prove this presumption. Based on the correlation between the presence and type of optic canal fracture and the visual prognosis in TON deduced from this study, we can design more logical and precise clinical trials which will help uncover this puzzle.

In conclusion, we found that $20.9 \%$ OCF cases, particularly linear intracanalicular fractures, were missed out on HRCT imaging. The presence and type of OCF might be a factor of visual prognosis for patients with TON. This should be noticed when reviewing HRCT scans of patients with TON and estimat-

Acknowledgements The authors thank doctors Xiaopeng Wang, Qiao Kong, Songping Yu, Fangzheng Jiang, Yan Chen, Wentao Jing and Linlin Chen for providing medical care for the patients and collecting the clinical data.

Contributors WW: design, analysis, writing and final approval; WY: design, data collection, writing the paper, analysis of data and final approval; YC: design, analysis, writing the paper, revising the paper and final approval. ZQ: design, analysis, drafting and final approval; DS: data collection, writing and final approval; PSC: analysis, writing and final approval; PD: analysis, writing and final approval; YT: image interpretation and final approval. ing the prognosis of patients. 
Funding This study was supported by the Natural Science Foundation of China (81371028), Natural Science Foundation of Zhejiang Province, PR China (LY12H12006) and the Guided Innovation Project of the Eye Hospital of Wenzhou Medical University (YNCX201104).

Competing interests None declared.

Patient consent Obtained.

Ethics approval The tenets of the Declaration of Helsinki were observed for this retrospective comparative case series, and the study was approved by the eight hospitals' ethics committees, which include the Eye Hospital of Wenzhou Medical University, the Central Hospital of Lishui, the Central Hospital of Jinhua, Lihuili Hospital in Ningbo, Quzhou People's Hospital in Zhjiang province, Xiamen Eye Hospital in Fujian province, The Fourth Hospital of Shengyang in Liaoning province, and The Second People's Hospital of Zhengzhou city in Henan province.

Provenance and peer review Not commissioned; externally peer reviewed.

\section{REFERENCES}

1 Anderson RL, Panje WR, Gross CE. Optic nerve blindness following blunt forehead trauma. Ophthalmology 1982;89:445-55.

2 Lee AG. Traumatic optic neuropathy. Ophthalmology 2000;107:814.

3 Steinsapir KD, Goldberg RA. Traumatic optic neuropathy: an evolving understanding. Am J Ophthalmol 2011;151:928-33.

4 Mauriello JA Jr, Lee HJ, Nguyen L. CT of soft tissue injury and orbital fractures. Radiol Clin North Am 1999;37:241-52.

5 Dietrich U, Feldges A, Nau HE, et al. Computed tomographic assessment of orbital fractures in traumatic damage of the optic nerve. Rofo 1990;152:185-90.

6 Seiff SR, Berger MS, Guyon J, et al. Computed tomographic evaluation of the optic canal in sudden traumatic blindness. Am J Ophthalmol 1984;98:751-5.

7 Guyon JJ, Brant-Zawadzki M, Seiff SR. CT demonstration of optic canal fractures. Am J Roentgenol 1984;143:1031-4.

8 Kubal WS. Imaging of the orbital trauma. Radiographics 2008;28:1729-39.

9 Lee HJ, Jilani M, Frohman L, et al. CT of orbital trauma. Emerg Radiol 2004; 10:168-72.

10 Unger JM. Orbital apex fractures: the contribution of computed tomography. Radiology 1984;150:713-17.

11 Cole $\mathrm{P}$, Kaufman $\mathrm{Y}$, Hollier L. Principles of facial trauma: orbital fracture management. J Craniofac Surg 2009;20:101-4.

12 Bodanapally UK, Van der Byl G, Shanmuganathan K, et al. Traumatic optic neuropathy prediction after blunt facial trauma: derivation of a risk score based on facial CT findings at admission. Radiology 2014;272:824-31.

13 Ramsay JH. Optic nerve injury in fracture of the canal. Br J Ophthalmol 1979;63:607-10.
14 Yang QT, Zhang GH, Liu X, et al. The therapeutic efficacy of endoscopic optic nerve decompression and its effects on the prognoses of 96 cases of traumatic optic neuropathy. J Trauma Acute Care Surg 2012;72:1350-5.

15 Imachi Y. Clinical and pathohistological investigations of the optic nerve lesions caused by head injuries. Nippon Ganka Gakkai Zasshi 1967;71:1874-908.

16 Yu-Wai-Man P, Griffiths PG. Surgery for traumatic optic neuropathy. Cochrane Database Syst Rev 2005;19:CD005024.

17 Gupta AK, Gupta AK, Gupta A, et al. Traumatic optic neuropathy in pediatric population: early intervention or delayed intervention? Int J Ped Otorhinolaryngol 2007;71:559-62

18 Wohlrab TM, Maas S, de Carpentier JP. Surgical decompression in traumatic optic neuropathy. Acta Ophthalmol Scand 2002;80:287-93.

19 Mine S, Yamakami I, Yamaura A, et al. Outcome of traumatic optic neuropathy. Comparsion between surgical and nonsurgical treatment. Acta Neurochir 1999;141:27-30.

20 Wang BH, Robertson BC, Girotto JA, et al. Traumatic optic neuropathy: a review of 61 patients. Plast Reconstr Surg 2001;107:1655-64.

21 Cook MW, Levin LA, Joseph MP, et al. Traumatic optic neuropathy: a meta-analysis. Arch Otolaryngol Head Neck Surg 1996;122:389-92.

22 Chou PI, Sadam AA, Chen YC, et al. Clinical experiences in the management of traumatic optic neuropathy. Neuro-ophthalmology 1996;16:325-36.

23 Tandon D, Thakar A, Mahapatra AK, et al. Trans-ethmoidal optic nerve decompression. Clin Otolaryngol Allied Sci 1994;19:98-104.

24 Li KK, Teknos TN, Lai A, et al. Traumatic optic neuropathy: result in 45 consecutive surgically treated patients. Otolaryngol Head Neck Surg 1999;120:5-11.

25 Chen CT, Huang F, Tsay PK, et al. Endoscopically assisted transconjunctival decompression of traumatic optic neuropathy. J Craniofac Surg 2007;18:19-26.

26 Levin LA, Beck RW, Joseph MP, et al. The treatment of traumatic optic neuropathy: the International Optic Nerve Trauma Study. Ophthalmology 1999;106:1268-77.

27 Chen C, Selva D, Floreani S, et al. Endoscopic optic nerve decompression for traumatic optic neuropathy: an alternative. Otolaryngol Head Neck Surg 2006;135:155-7.

28 Chen F, Zuo K, Feng S, et al. A modified surgical procedure for endoscopic optic nerve decompression for the treatment of traumatic optic neuropathy. N Am J Med Sci 2014;6:270-3

29 Levin LA, Joseph MP, Rizzo JF, et al. Optic canal decompression in indirect optic nerve trauma. Ophthalmology 1994;101:566-9.

30 Lu Y, Pan J, Qi S, et al. Pneumatization of the sphenoid sinus in Chinese: the differences from Caucasian and its application in the extended transsphenoidal approach. J Anat 2011;219:132-42.

31 Kantarci M, Karasen RM, Alper F, et al. Remarkable anatomic variations in paranasal sinus region and their clinical importance. Eur J Radiol 2004;50:296-302. 